\title{
Identification of Preferred Product Image under the Visual Cognitive Neural Mechanism
}

\author{
Yang Chen ${ }^{1}$, Li Lin*², Zhiang Chen ${ }^{2}$ \\ ${ }^{1}$ Key Laboratory of Advanced Manufacturing Technology, Ministry of Education, \\ Guizhou University, Guizhou, China \\ ${ }^{2}$ School of Mechanical Engineering, Guizhou University, Guizhou, China \\ linlisongbai@163.com
}

Keywords: Eye-tracking technology, EEG technology, perceptual image, preferred image

\begin{abstract}
In order to improve the objectivity of the measurement of preferred product image, eye-tracking combined with EEG technology was used to locate users' preferred image of products. The method included the following steps: (1) Product samples and image words of the subjects as stimuli materials were imported into the computer's picture viewing module, which displayed the product samples or image words; (2) Participants watched the stimuli materials; (3) Eye movements and EEG data were collected by telemetry eye-tracker and electroencephalograph according to the reaction of participants when they watched the stimuli materials; (4) Preferred image was obtained by comprehensive evaluation of the average gaze duration of eye movements data and the asymmetry index of frontal alpha of EEG data. The experimental results show that the image words of "Complex-Concise”, "Retro-Modern”, "Smart-Heavy”, "Lively-Serious" caused participant's average gaze duration to be shorter than other words. Moreover, the power of alpha waves captured by the left frontal channel (F3) was significantly lower than the average power of the alpha waves captured by the right frontal channel (F4) when participants observed the image words of "Concise", "Gorgeous", "Modern", "Smart", "Lively". It shows that user's preferred image of perceptual products can be obtained more scientifically and effectively by using the combined physiological measurement technologies of eye-tracking and EEG, and reference can be provided for design applications based on user's preferred image in silver jewelry.
\end{abstract}

\section{Introduction}

The perceptual image presented by product's appearance in human brain is the most direct feeling that the product gives to us, which can express people's values and preferences for the contemporary environment [1]. Perceptual image can be recognized because it is represented in the form of reflecting the characteristics of certain cultural and social backgrounds [2]. Since a user's expression of product's image reflects his/her perceptual preferences for the product, understanding consumer's preferences for image is a key for companies to manufacture products that meet user's psychological expectations and promote consumptions, while can help designers improve their 
design capabilities. In the existing research on product's image, psychometric methods such as semantic difference (SD) method are widely used to obtain perceptual image that conform to consumer's preferences ([3], [4], [5]). Although SD method and other methods are extremely flexible and easy to be conceived, it is difficult to identify the real and accurate psychological state of users by using a single psychological measurement method because the expressions of preferred image are vague and incomplete.

Along with the development of neuroscience and brain science, the physiological measurement techniques that scientific instruments are used to measure and record user's psychological reactions have been increasingly applied in the fields of marketing and product design. From the perspective of the visual cognitive neural mechanism, Khushaba et al. studied the formation of user's preferences, the formulation of purchasing decisions, the product's evaluation and design application ([6], [7], [8], [9]). In view of the fact that it's difficult to fully express preferences when people are explicitly asked, the physiological measurement methods have been adopted to "deliberately" reveal hidden information about consumer's preferred image from the origin of human cognition and turn it into the information of physiological activities that cannot be provided by conventional psychological measurement methods [6]. It is an inevitable trend for in-depth implementation of user-driven design in the future.

Under the visual cognitive neural mechanism, vision is the main channel to obtain information of products, and directly affects user's preferences and purchase intentions [10]. A large number of studies have used eye-tracking method to acquire user's perceptual preferences, Kostyra et al. used eye-tracking technology combined with semantic scale to study the sensory characteristics of smoked ham and evaluate consumer's sensory preferences [11]. Colombo et al. used eye-tracking technology and self-report questionnaire evaluation method to explore how specific elements in the interior environment design affected user's preferences and purchase intentions [12].

EEG is a useful tool for studying the relationship between brain activity and emotional state ([13], [14]), so consumer's brain waves can be used to analyze and judge their preferences. Researchers generally believed that the medial prefrontal lobe mainly characterizes consumer's preferences and value assessments of product and has a function of purchasing prediction, the orbitofrontal lobe mainly characterizes consumer's pleasant experience and purchase intentions [15]. The results of Khushaba et al. 's study showed that preferred objects can cause significant changes in power spectrum activity on frontal lobe (channel F3 and F4) [16], the electrode profile is shown in Figure 1. Briesemeister et al. found that, according to the frontal alpha asymmetry, the frequency domain characteristics of the alpha waves from left and right frontal can be used to learn the emotional characteristics of user [17], which can help designer obtain information of user's preferences by learning user's emotions [18]. Hsu et al. proposed a method of comprehensively using psychological tasks and EEG data to detect user's emotions [19].

In addition, Gao X Q et al. believed that the combination of eye-tracking technology and EEG technology can further understand the mechanism of an individual's cognitive activities [20]. Yang M Q et al. proposed a method of affective pictures classification based on information about the user's experience collected through measurable eye movements and EEG signals [8]. Khushaba et al. used eye-tracking and EEG technology to study the physiological decision-making process of consumer, observed and evaluated the cortical activities in different brain regions when they make 
preferred selection for product [6]. Li S et al. established a relationship model between the experimental indexes of eye-tracking and EEG and PAD emotional state values with shopping websites as the objects of study [21]. Tang B B et al. proposed a cognitive evaluation method for aesthetic experience of the automobile industrial design combining eye-tracking and EEG technology [22].

These studies have laid foundations for measurement and evaluation of perceptual information in the perspective of the visual cognitive neural mechanism. With the deepening of user-driven design, the acquisition of user's preferred image which is an important premise of perceptual product design can provide possibilities for designing product that is closer to the user's appeals. Therefore, based on the theory of cognitive neuroscience and cognitive psychology, advantages of eye-tracking and EEG technology in cognitive research were made full use of, the cognition of preferred image was reflected by eye-brain data from the perspective of the visual cognitive neural mechanism, the deep hidden design information from user was explored and revealed from the source of cognition, which provides reliable data and scientific basis for designer to obtain user's preferred image more accurately.

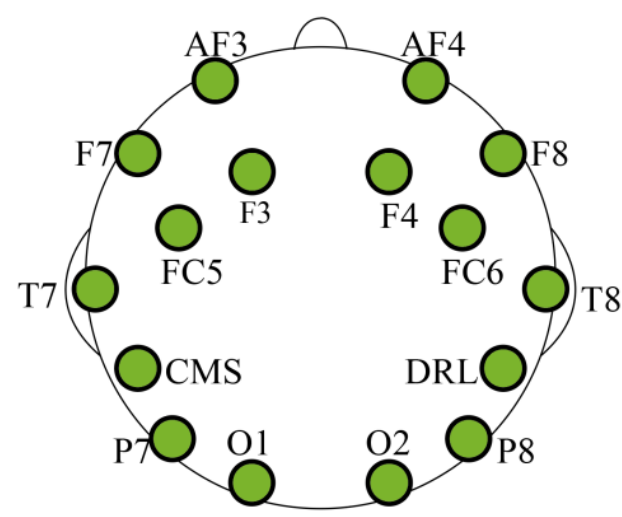

Figure 1. 14 channel electrode profile on Emotive EPOC+

\section{Measurement of user's preferred image for silver earpieces with eye-tracking and EEG technology}

Under the guidance of cognitive neuroscience theory and principle of visual perception, the changes of eye movements and brain waves when user made a simple matching (decision-making) were explored to obtain user's preferences for test samples from the eye-brain cognitive data, and image adjectives were used as the representations of preferences. The matching situation was designed as user's judgments of the image adjectives that they thought could describe the product in accordance with their preferences. The image adjectives that they thought fit their preferred samples were the image adjectives that that match their preferences. Taking the samples of silver earpieces as an example, the measurement processes of the preferred image with eye-tracking combined with EEG are shown in figure 2. First, user preferred pictures and image words were made into test materials, which were presented to subjects through programming with e-prime software. The participant's eye-movement data were recorded by SMI eye-tracker, the changes of EEG signals were recorded by Emotive EPOC+. 


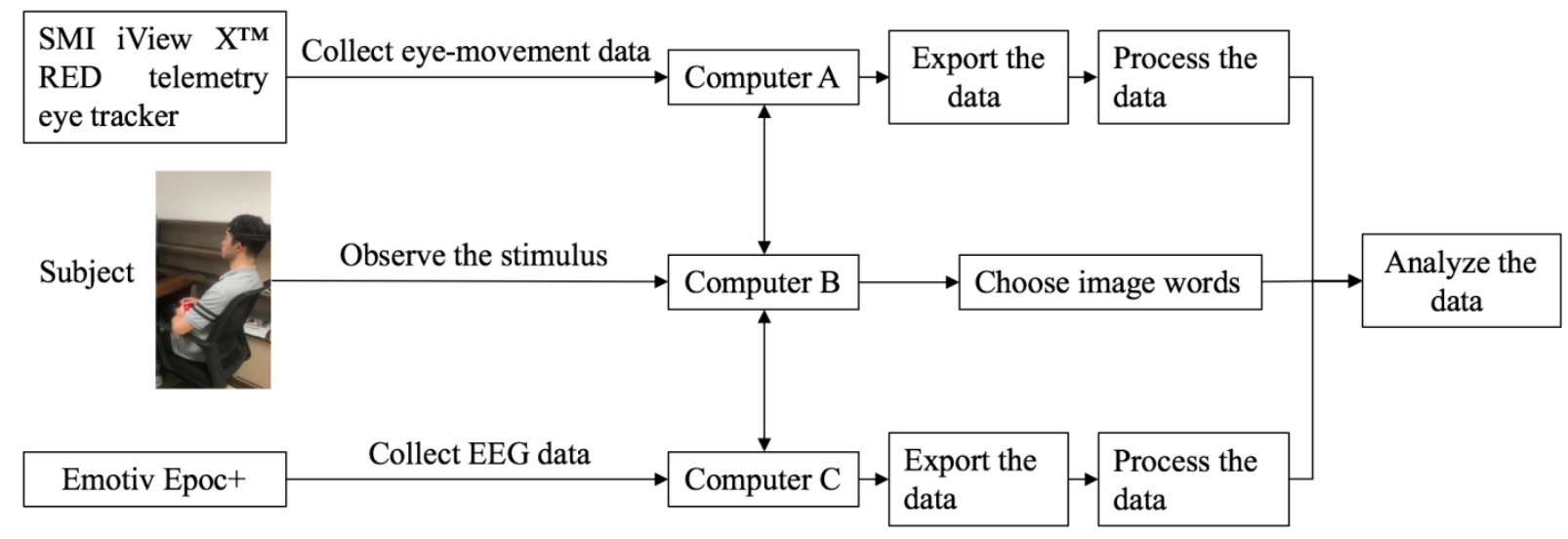

Figure 2. Flow of measurement

\subsection{Preparation of stimuli materials}

Firstly, 150 pictures of silver earpieces were preliminarily obtained. Through subjective comparative analysis, pictures with obvious similarity or repetition were screened, and 100 pictures were retained. After multivariate scale analysis and clustering analysis, 16 typical pictures were finally selected, as shown in figure 3. Typical samples were divided into many groups, 4 for each group, arranged by the Latin square method. Eye tracking technology was used to screen the preferences of 16 samples, and average gaze duration and average gaze frequency were used as the analysis indexes of eye movements to obtain 8 samples conforming to user's preferences, as shown in figure 4.
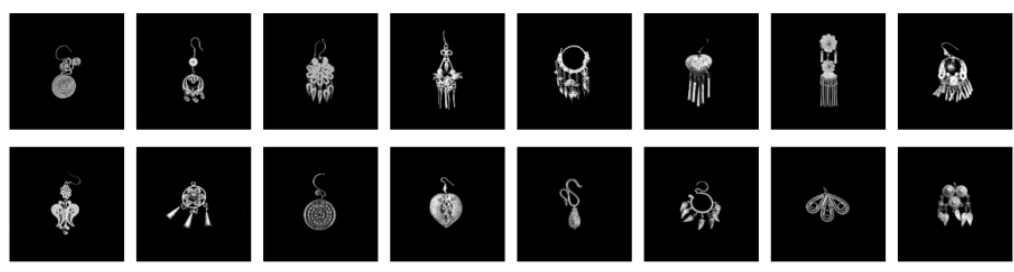

Figure 3. Typical samples

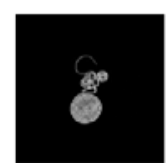

1

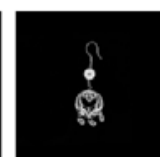

2

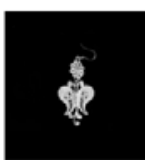

3

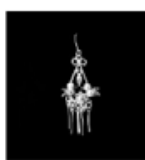

4

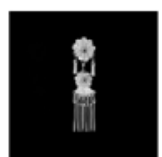

5

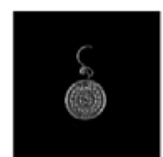

6

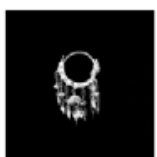

7

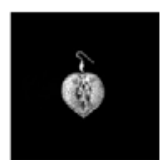

8

Figure 4. Samples conform to user's preferences

A total of 200 perceptual adjectives about silver earpieces were collected. Ambiguous, similar and obscure image words were removed, then the artificial hierarchical clustering method was used. 58 pairs of perceptual image words were obtained by matching the adjectives with opposite meanings. Then the questionnaire method was used to further screen with the help of participants. Finally, the number of pairs of perceptual image words was reduced to 5 pairs, as shown in table 1 . 
Table 1. Image words pairs

\begin{tabular}{|c|c|c|c|c|c|}
\hline No. & $\mathbf{1}$ & $\mathbf{2}$ & $\mathbf{3}$ & $\mathbf{4}$ & $\mathbf{5}$ \\
\hline $\begin{array}{c}\text { Image words } \\
\text { pairs }\end{array}$ & Complex-Concise & Gorgeous-Plain & Retro-Modern & Smart-Heavy & Lively-Serious \\
\hline
\end{tabular}

\subsection{Measurement Experiment of User's Preferred Image with Eye-tracking and EEG Technology}

\subsubsection{Participants}

In this study, 18 consumers of silver ornaments were selected from Guizhou University. The ratio of male to female was $1: 1$. All participants were between the ages of 20 and 40 (the mean age is 27). Participants were asked to wash their hair to reduce the resistance to scalp grease before the experiment. There were some rewards after the experiment.

\subsubsection{Experimental equipment and environment}

In this study, wireless multi-channel EEG system Emotive EPOC+ was used to collect EEG data. It consists of 14 channels with two additional reference electrodes located behind the ear. Figure.1 shows the distribution of electrode positions. Each EEG channel is recorded at a sampling frequency of $128 \mathrm{~Hz}$. Meanwhile, RED desktop eye tracker from SMI Company in Germany was used to collect eye movements' data. With e-prime, psychological programming software, the two devices were synchronously measured.

The experiment was carried out in the Institute of Kansei Engineering, Guizhou university. During the experiment, there was no other person or noise interference except experimenter and participant. The indoor temperature and humidity were kept at an appropriate level to feel comfortable.

\subsubsection{Experimental procedures}

(1) Participant (subject) enters the laboratory to familiarize himself/herself with the experimental environment and equipment, registers personal information, and takes a rest to calm down.

(2) Experimenter introduces the process of eye-tracking combined with EEG experiment and precautions to the subject.

(3) Subject wears and calibrates the instruments.

(4) Experimenter starts the experimental procedure and subject reads the instructions.

(5) The pre-experiment starts running, subject observes the pre-experimental materials, then uses left or right button on the mouse to select whether the image words meet the description of the samples. The left button indicates "match" and the right button indicates "no match".

(6) The experiment starts running, subject observes the experimental materials, and uses left or right button on the mouse to select whether the image words meet the description of the samples. During the experiment, the experimenter controls and observes the collection of physiological indexes such as gaze, saccade, and EEG.

(7) At the end of the experiment, instruments stop recording.

The experimental procedures are shown in Figure 5. (Note: In order to ensure the effectiveness of experimental results, subjects should be familiar with the positions of left and right buttons on mouse in advance, so as to avoid bow down or look away when making selection.) 


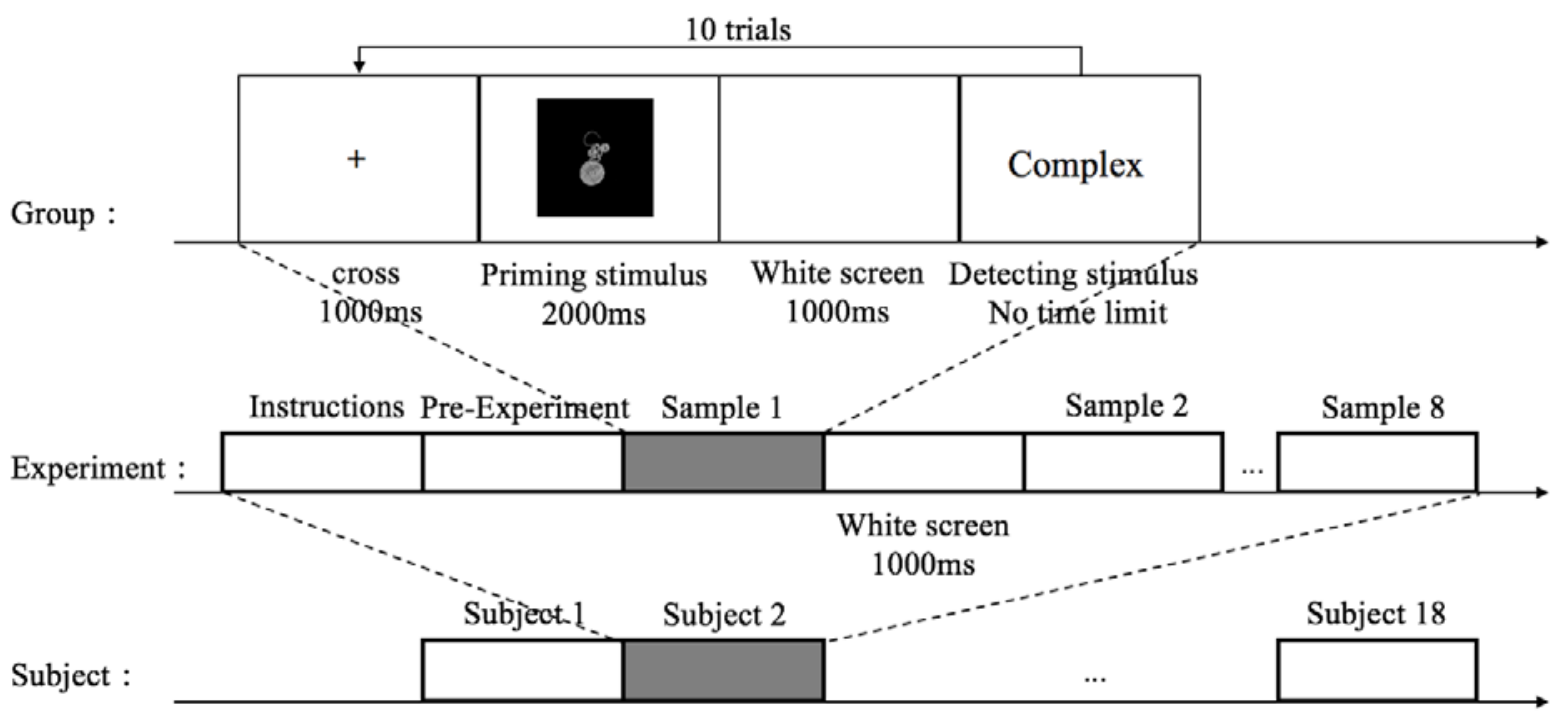

Figure 5. The experimental procedures

\section{Data processing}

\subsection{Selecting the indexes for Eye Movement and EEG measurement}

There is a significant correlation between fixation duration in the eye movement index and preference ([23], [24]). When the product-image matching result is definite, the gaze duration is shorter, when the product-image matching result is fuzzy, the thinking time is prolonged and the gaze duration is significantly increased ([25], [26]). So, the average gaze duration [ms] was selected as the index of eye-movement to analyze the user's preferences for earpieces.

For brain cognition, cerebral cortex mainly divided into the frontal, parietal, occipital and temporal lobe, each has a different functional division of labor. Among them, the frontal lobe is an important area of processing cognitive information [27]. Therefore, F3 (left frontal lobe) and F4 (right frontal lobe) were selected as the main channels to obtain brain cognitive information. The common rhythm waves in EEG are delta $(\delta, 1 \sim 4 \mathrm{~Hz})$, theta $(\theta, 4 \sim 8 \mathrm{~Hz})$, alpha $(\alpha, 8 \sim 13 \mathrm{~Hz})$, beta ( $\beta, 13 \sim 30 \mathrm{~Hz})$ and gamma $(\gamma, 30 \sim 45 \mathrm{~Hz}) . \alpha$ is thought to be involved in the processing of emotions [17]. The left and right hemispheres of human brain differ in structure and function. They lead different types of emotion processing, while the right hemisphere is mainly responsible for the processing of negative emotions and the left hemisphere is mainly responsible for the processing of positive emotions [28]. The relationship between $\alpha$ waves and emotions is mainly reflected in the asymmetry between the left and right hemispheres of the frontal region, which is often measured by the asymmetry index of frontal alpha, that is, the power of $\alpha$ waves captured by F4 minus the power of $\alpha$ waves captured by F3 [29]. Therefore, the asymmetry index of frontal alpha was selected as the index of EEG to characterize the preferred image of silver earpieces.

\subsection{Processing the indexes for Eye Movement and EEG measurement}

The collected eye movements' data were analyzed and processed by BeGaze analysis software.

The main steps of processing the collected EEG signals include: 1) pre-processing: the doped artifacts in the collected EEG signals were removed by filtering and independent component analysis; 2) EEG data segmentation: from 500ms before the emergence of each image word as the detecting stimulus to $1000 \mathrm{~ms}$ after its emergence, a total of $4500 \mathrm{~ms}$ was used as an analysis period; 3) feature extraction: the power spectral density was calculated, Fourier transform was used to map the original EEG signal to $\alpha$ band, and Alpha band power spectrum was calculated. EEG power 
spectrum was obtained from the integration of EEG power spectrum density [30]:

Frequency Resolution: $d=\frac{1}{4.096 s}$

$$
\mathrm{PS}_{(\alpha)}=\sum_{w 1}^{w 2} \mathrm{p}(\mathrm{w}) * \mathrm{~d}
$$

$\mathrm{PS}_{(\alpha)}$ : The power of alpha waves

$w 1, \quad w 2$ : The upper and lower limits of frequency band

The asymmetry index of frontal alpha [29] = power of $\alpha$ waves captured by F4 - power of $\alpha$ waves captured by F3

\section{Results}

\subsection{Eye-movement data}

The average gaze duration obtained by eye-tracker is evaluated as shown in Table 2. When the product-image matching results are definite, that is, the subject confirms that those image words match (not match) the descriptions of the product, the average gaze duration of the subject is shorter. When the product-image matching results are fuzzy or there are no obvious affective preferences, the average gaze duration is longer.

Table 2. The average gaze duration of 5 image words pairs (8 samples of silver earpieces)

\begin{tabular}{|c|c|c|c|c|c|c|c|c|c|c|}
\hline \multirow{2}{*}{ image words } & \multicolumn{2}{|c|}{$\mathbf{1}$} & \multicolumn{2}{|c|}{$\mathbf{2}$} & \multicolumn{2}{|c|}{$\mathbf{3}$} & \multicolumn{2}{c|}{$\mathbf{5}$} \\
\cline { 2 - 10 } & Complex & Concise & Gorgeous & Plain & Retro & Modern & Smart & Heavy & Lively & Serious \\
\hline $\begin{array}{c}\text { average gaze } \\
\text { duration } \\
(\mathbf{m s})\end{array}$ & 557.32 & 549.02 & 723.16 & 714.50 & 593.51 & 590.55 & 543.81 & 522.42 & 556.86 & 590.40 \\
\hline
\end{tabular}

The experimental results show that when subject made matching judgments on the five pairs of image words, Group 1, 3, 4 and 5 evoke shorter average gaze duration, and the results indicate that most subjects are more efficient in matching "Complex-Concise "Retro-Modern", "Smart-Heavy", "Lively-Serious", that is, most subjects make it clear whether these image words are consistent with the description of the product, but the positive and negative emotions of subjects cannot be distinguished. The average gaze duration of the Group 2 is significantly longer than that of the other groups, indicating that subjects do not have obvious emotional bias toward the matching of "gorgeous-plain" image words and product, more cognitive processing resources need to be allocated to judge it. Since the user's preferences in implicit cognitive process is composed of preference tendency and preference degree, eye-movement data alone cannot draw conclusions about preference tendency, which needs to be further confirmed with EEG data.

\subsection{EEG data}

The preference-related characteristic variables of EEG generated by subject in making judgments are used to calculate the changes of EEG spectral activities. The asymmetry index of frontal alpha can be used to judge the emotional state of subjects and obtain the preferences of participant from emotions. The greater the asymmetry index of frontal alpha, the more the image words fits user's preferences.

Table 3. the asymmetry index of frontal alpha of 5 image words pairs (8 samples of silver earpieces)

\begin{tabular}{|c|c|c|c|c|c|c|c|c|c|c|}
\hline \multirow{2}{*}{ image words } & \multicolumn{2}{|c|}{$\mathbf{1}$} & \multicolumn{2}{|c|}{$\mathbf{2}$} & \multicolumn{2}{|c|}{$\mathbf{3}$} & \multicolumn{2}{|c|}{$\mathbf{4}$} & \multicolumn{2}{|c|}{$\mathbf{5}$} \\
\cline { 2 - 10 } & Complex & Concise & Gorgeous & Plain & Retro & Modern & Smart & Heavy & Lively & Serious \\
\hline $\begin{array}{c}\text { asymmetry index of } \\
\text { frontal alpha }\end{array}$ & -0.011 & 0.053 & 0.037 & -0.012 & -0.023 & 0.038 & 0.055 & -0.017 & 0.058 & -0.025 \\
\hline
\end{tabular}


The asymmetry index of frontal alpha during the matching experiment is shown in Table 3. The experimental results show that, when subjects make matching judgments for five pairs of image words, "Concise", "Gorgeous", "Modern", "Smart", "Lively" than "Complex", "Plain", "Retro", "Heavy", "Serious" induce greater asymmetry index of frontal alpha, indicating that subjects are more likely to generate positive emotions when judging whether the above image words can describe the preferred samples, which further verify that the above image words are more consistent with user's cognitive preferences.

\section{Conclusion}

By observing the subjective evaluation results and eye movements data and EEG signals recorded during the stimulation process, from the visual cognitive neurological perspective of eyetracking combined with EEG technology, consumer's preferred image words of product are obtained. For silver earpieces in this study, eye movements results show that users have obvious emotional bias towards image words such as "Complex-Concise", "Retro-Modern", "Smart-Heavy", "LivelySerious", and have certain cognitive ambiguity towards "Gorgeous-Plain". EEG results show that "Concise", "Gorgeous", "Modern", "Smart" and "Lively" are more likely to induce positive emotions, However, as a result of eye movements measurement, it is known that user's emotional preferences for the matching of "Gorgeous" and silver earpieces are less obvious, that is, user's preferred image for silver earpieces is "Concise", "Modern", "Smart" and "Lively". eye-tracking combined with EEG technology can be used as an effective method to measure user's perceptual preferences for image. The results of eye movements and EEG research can lay a foundation for measurement of perceptual information and its manifestation from the perspective of the visual cognitive neural mechanism, a scientific and effective measurement method of user's preferred image for product design based on user's cognitive preferences is provided, and a reference is provided for the researches on the design applications based on user's preferred image for silver earpieces.

There are still some limitations in this study. In the process of preference selection, each subject's EEG data had great difference, which leaded to the reliability of EEG data is insufficient. In order to verify the results, more subjects and a better age distribution are needed to improve the reliability, validity and universality of the results.

\section{Acknowledgements}

This project is supported by National Natural Science Foundation of China [Grant No. 51465007 and 51865003].

\section{References}

[1] Sun Q. Research on Product Modeling Method Based on Imagery. 2007.

[2] Chan C S. Can style be measured? Design Studies, 21: 277 - 291,2000.

[3] Ming C C, Chang C C and Shang H H. Perceptual factors underlying user preferences toward product form of mobile phones. International Journal of Industrial Ergonomics, 27: 247-258, 2001.

[4] Wei C C, Ma M Y and Lin Y C. Applying Kansei Engineering to Decision Making in Fragrance Form Design. Intelligent Decision Technologies, 10:85 - 94,2011.

[5] Han J X and Ma M Y. A Study on Developing the Kansei Engineering of Jewellery in Female Users: Take PANDORA Jewellery for Example. International Conference on Kansei Engineering \& Emotion Research, 94 - 103, 2018.

[6] Khushaba R N, Wise C and Kodagoda S. Consumer neuroscience: Assessing the brain response to marketing stimuli using electroencephalogram (EEG) and eye tracking. Expert Systems with Applications. 40: 3803 - 3812, 2013. 
[7] Kenning P H and Plassmann H. How neuroscience can inform consumer research? IEEE Transactions on Neural Systems and Rehabilitation Engineering, 16: 532 - 538, 2008.

[8] Yang M Q, Lin L and Milekic S. Affective Image Classification Based on User Eye Movement and EEG Experience Information. Interacting with Computers, 30:417 - 432, 2018.

[9] Zhou Z Y, Cheng J X and Wang S A. Current Situation and Trend of EEG Research in Industrial Design, Packaging Engineering, 39: 219 - 223, 2018.

[10] Moshagen M and Thielsch M. Facets of visual aesthetics. International Journal of Human-Computer Studies. 68: $689-709,2010$.

[11] Kostyra E, Wasiak-Zys G and Rambuszek M. Determining the sensory characteristics, associated emotions and degree of liking of the visual attributes of smoked ham. A multifaceted study. LWT-Food Science and Technology, 65: $246-253,2016$.

[12] Colombo B, Laddaga $S$ and Antonietti A. Psychology and design. The influence of the environment's representation over emotion and cognition. An ET study on Ikea design. Procedia Manufacturing, 3: 2259 - 2266, 2015.

[13] Lee Y Y and Hsieh S. Classifying different emotional states by means of EEG-based functional connectivity patterns. Plos One, 9: e95415, 2014.

[14] Bos D O. EEG-based Emotion Recognition. The Influence of Visual and Auditory Stimuli. Emotion, $1359: 667$ 670, 2012.

[15] Guo F, Ding Y and Wang T. Applying event related potentials to evaluate user preferences toward smartphone form design. International Journal of Industrial Ergonomics, 54: 57 - 64, 2016.

[16] Khushaba $R \mathrm{~N}$, Greenacre $L$ and Kodagoda S. Choice modeling and the brain: A study on the Electroencephalogram (EEG) of preference. Expert Systems with Applications, 39: 12378 - 12388, 2012.

[17] Briesemeister B B, Tamm S and Heine A. Approach the good, withdraw from the bad-A review on frontal alpha asymmetry measures in applied psychological research. Psychology, 4: 261 - 267, 2013.

[18] Deng L, Chen B and Pang Q Y. Application of EEG Technology in Emotional Design of Cultural Creative Products. Journal of Graphics, 39: 327 - 332, 2018.

[19] WY Hsu. An integrated-mental brainwave system for analyses and judgments of consumer preference[J]. Telematics and Informatics, 2017, 34(5):518 - 526.

[20] Gao X Q, Wang Y Y and Ge L Z. The Combination of Eye Movement Technique and EEG Technique-A New Approach to Cognitive Research. Ergonomics,11:36 - 38, 2005.

[21] Li S, Zhuang X X and Liu W L. Study on emotion measurement method of EEG and eye movement technology fusion. IE\&M, 12: $144-148,2014$.

[22] Tang B B, Guo G and Wang K. Combined with eye movement and EEG, the auto industry design user experience selection. Computer Integrated Manufacturing Systems, 6:1449 - 1459, 2015.

[23] Liu S Q, Liu B Y and Li N. Middle school students' preference for multimedia browsing behavior and its teaching value - an experimental study on eye movement based on multimedia interface structure. E-education Research, 7: $114-116,2018$.

[24] Jantathai S, Danner L and Joech M. Gazing behavior, choice and color of food: Does gazing behavior predict choice? Food Research International, 54:1621 - 1626, 2013.

[25] Yang C, Chen $C$ and Tang Z C. Research on product image reasoning model based on EEG. Journal of Mechanical Engineering, 2017

[26] Guo F, Qu Q X and Zhang X Y. Study on the relationship between user eye movement behavior and web design elements. IE\&M, 19:129 - 139, 2014.

[27] Costa T, Rognoni E and Galati D. EEG phase synchronization during emotional response to positive and negative film stimuli Neuroscience Letters, 406:159 - 164, 2006.

[28] He L J. Emotional response and recognition under different acoustic stimuli. 2013.

[29] Coan J A and Allen J J B. Frontal EEG Asymmetry as A Moderator and Mediator of Emotion. Biological Psychology, 67: 7 - 49, 2004.

[30] $\mathrm{Lu} \mathrm{Y} \mathrm{J,} \mathrm{Dai} \mathrm{L} \mathrm{L} \mathrm{and} \mathrm{Wu} \mathrm{H} \mathrm{Z.} \mathrm{EEG} \mathrm{study} \mathrm{on} \mathrm{the} \mathrm{soothing} \mathrm{effect} \mathrm{of} \mathrm{different} \mathrm{types} \mathrm{of} \mathrm{music} \mathrm{on} \mathrm{sadness.}$ Psychological Exploration, 32:369 - 375, 2012.

[31] Chen M, Wang H Y and Xue C Q. Product image-semantic matching evaluation based on event-related potentials. Journal of Southeast University, 44: 58 - 62, 2014. 